\title{
Construction and Analysis of the Finite Element Model in the Chinese Bone Setting Training System
}

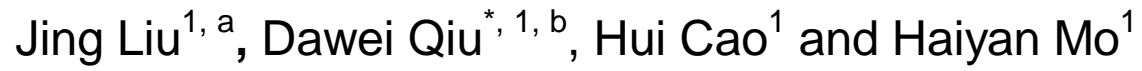 \\ ${ }^{1}$ College of Science and Engineer, Shandong University of Traditional Chinese Medicine, Jinan, \\ Shandong Province, China

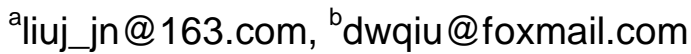

Keywords: Finite element model, Chinese bone setting, construction and analysis.

Abstract. The main contribution of the paper is to construct and analyze the finite element model in the Chinese bone setting training system. First we construct humeral geometrical model, based on this model we build up the finite element model. The experiments show the obtained models are efficient.

\section{Introduction}

Chinese bone setting has a long history and is a treasure of Traditional Chinese Medicine, it can cure many types of bone fractures without damaging bone tissue. It has many advantages such as healing quickly, low cost and better effect. Chinese bone setting is a clinical technique with strong practical nature, it can be mastered only with much training. At present, learning Chinese bone setting mainly depends on teachers' explaining, pictures and videos, and it is not realistic to let learners exercise on the patients for there is few clinical cases. Meanwhile, though the Chinese bone setting has a wide clinical utilization, but the research of biomechanics lagged relatively, it's hard to explain the principle of the Chinese bone setting scientifically, and there is no uniform technical specification and evaluation criteria, these influence heritages and developments of the Chinese bone setting.

Simulation-based medical education (SBME) was applied to modern medical education and showed the great superiorities and broad perspectives with the developments of science and technology and the transformations of medical patterns [1]. The so called SBME is a clinical teaching method used for simulated training through various simulation techniques to create similar real patients and scenarios [2]. The method can relieve the deficiency of clinical resources effectively and make the teaching conditions more objective and operable [3]. It can solve the problems above effectively that SBME used in the teaching of the Chinese bone setting.

In the paper, we first discuss how to construct the humeral geometrical model, then based on the model, we explore the construction of the finite element model. Experiments imposed different payload show the models are satisfied to the clinical teaching.

\section{Related Works}

The objectification of Chinese bone setting refers to in the manipulation process the strength, translation and trajectory of the sleight of hand were acquired and analyzed using scientific quantifiable methods and then guide and evaluate the medical manipulations. The biological mechanic properties of the Chinese bone setting consists of mechanics and kinetics. Mechanic characteristics include the magnitude of strength, direction, point of action, moment of force and momentum etc. kinetics ones include frequency, translation, velocity, trajectory etc.

Nan Geng etc. [4] analyzed mechanics parameters of the positioning and rotation of cervical vertebra. Liheng Wang etc. [5] selected 51 cases of cervical vertebra patients and obtained some data using massage, twisting and rotating manipulations. Professor Liguo Zhu [6] from Chinese Science Academy of Traditional Chinese Medicine constantly works on measurement, analysis and research of the Chinese manipulations, especially did more researches on biological mechanics characteristic and influence factors.

\footnotetext{
* Correspondence author
} 
In recent years, the finite element technique was frequently used in simulation model research, it is a valuable complement of biological mechanics characteristic for clinical research. The finite element technique was firstly used in engineering as a numerical analysis method. Its basic idea was partitioning a continuous elastic object into finite and small units that connecting to an aggregation, units transmit acting force, the mechanic performance of the entire object is solved by computing each unit. In 1972, the finite element technique was first used in bone biological mechanics research by Brekelmas [7] and Ryblcki [8]. The biological mechanics research of the four limbs bone based on the finite element technique mainly focused on the legs especially thigh-bone and hip joint. Fewer research refers to arms.

Medical 3D visualization research first developed from Europe and USA, in the late of $20^{\text {th }}$ century, USA proposed "visual human" project, and fulfilled the slice scanning of a male and female body in 1991 and 1994. With the rapid development of medical 3D reconstruction, people realized the importance of its clinical application. Some companies developed professional software libraries, such as VTK, ITK, RTVR and VGL etc.

\section{Construction of Humeral Geometrical Model}

In the section, we construct a humeral geometric model. First import data into the system, then through thresholding and region growing steps, at last we get the 3D model.

\section{Import Data}

MIMIC can accept automatic and manual many kinds of CT image formats. Because DICOM data sets include all required parameters, so we select automatic imported original DICOM. MIMIC can detects automatically the size of the image, pixel dimension, the center of the reconstruction and save to its own format. We import data into MIMIC.

\section{Thresholding}

Thresholding partitions the image into foreground and background through different gray level. In order to segment the target region effectively, we draw a line through the segmented target and set the threshold between 226 and 251 .

\section{Region growing}

Region growing is the method that merges those adjacent pixels or region with similar properties into a larger region by selecting a seed. In the paper, after thresholding, we select a pixel on the humerus, then run region growing, those pixels with similar gray values will be extract, because the top of ulna and radius is linked together with the humerus, so they are extracted too. The results are showed in Fig. 1

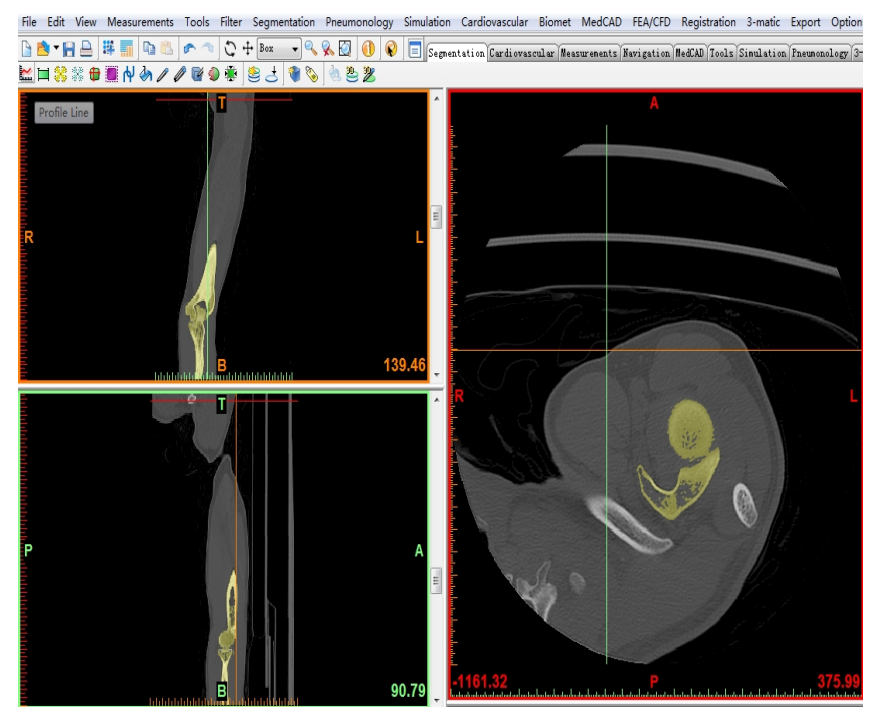

Fig. 1 region growing results 


\section{Build 3D model}

The prerequisite of generating 3D model is precise segmentation. In the paper, the initial 3D model is very coarse, there is some small burr, bump and sag, and this will burden the subsequent computation, after smoothing, the results before and after smoothing are showed in Fig. 2.

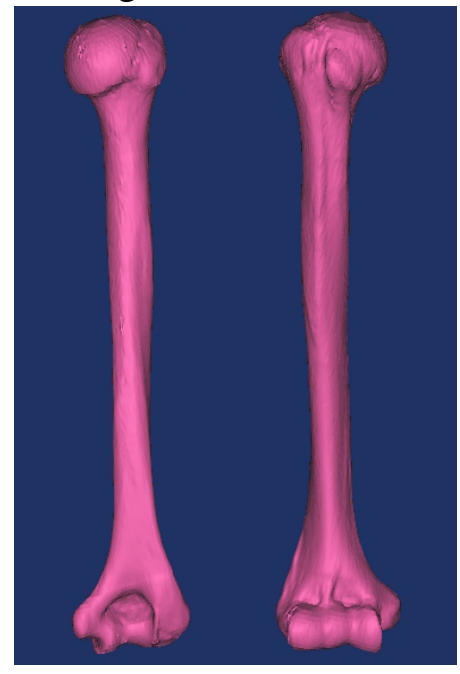

(a)

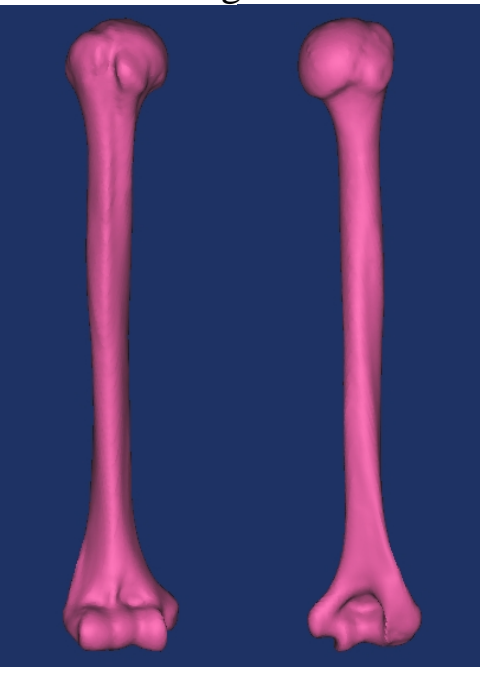

(b)

Fig. 2 (a) before smoothing. (b) after smoothing

\section{Construction of the Finite Element Model}

The above 3D geometric model need to be transformed into entity structure to build the finite element model, grid partition is the first step to obtain the model. After obtained grids, we compute the gray values of every unit, then set corresponding material according to different gray range, or according to density, elastic and Poisson ratio, then we can use these data in the finite element analysis. Fig. 3 showed the obtained the finite model. The final results after imposing 100N are showed in Fig. 4.

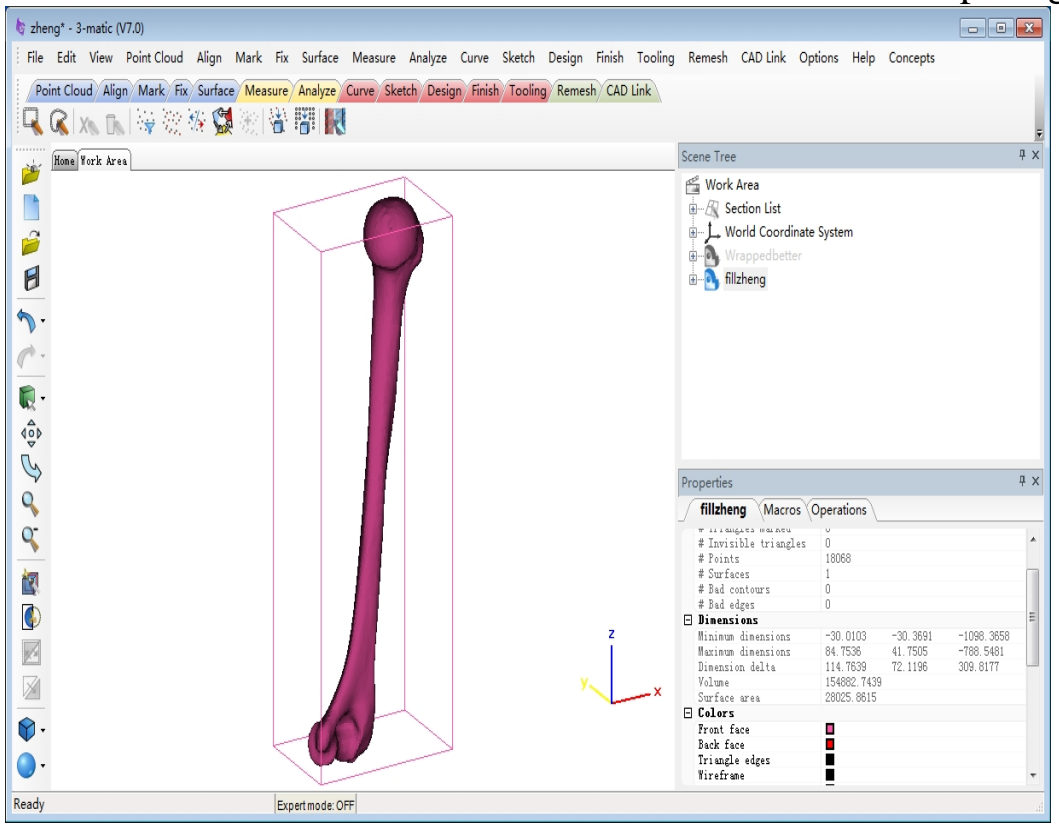

(a)

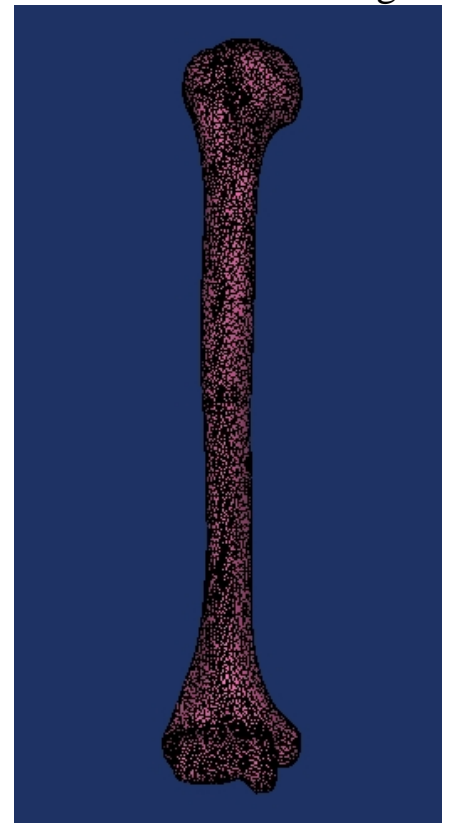

(b)

Fig. 3 the obtained finite element model 


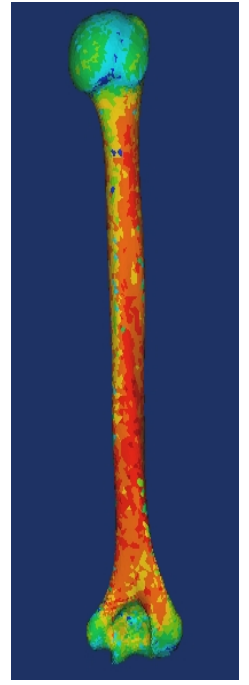

(a)

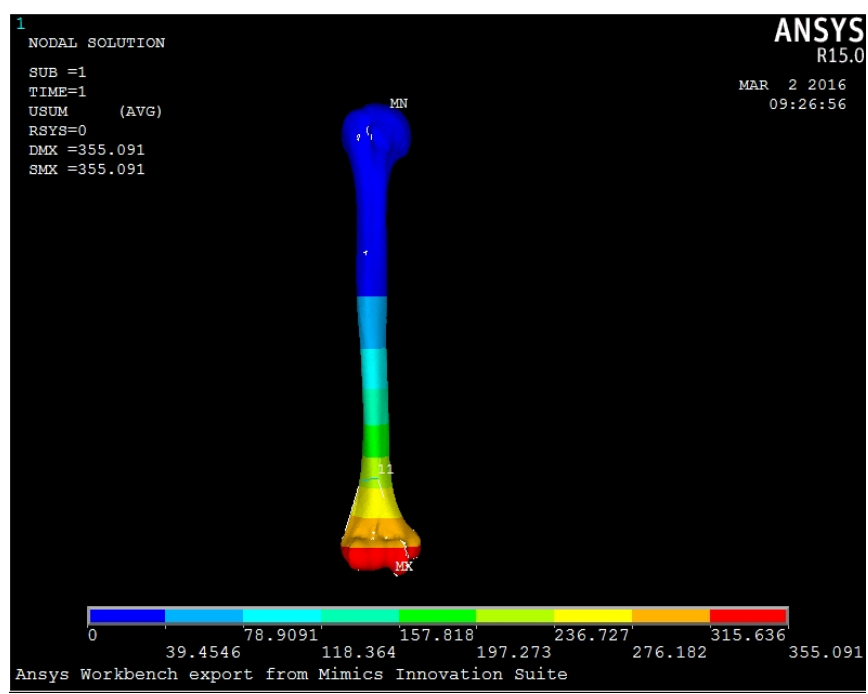

(b)

Fig. 4 (a) setting material for humerus (b) translation variable cloud fig of payload 100N

\section{Conclusions}

If you follow the "checklist" your paper will conform to the requirements of the publisher and facilitate a problem-free publication process.

\section{Acknowledgements}

This work was financially supported by the Science and Research Development Projects of Shangdong Province (J15LL04) and the National Natural Science Foundation (NO. 81473708).

\section{References}

[1] Liping Peng, Ningda Xu and Xiao Chen: The Simulation Teaching of Chinese bone setting. New Traditional Chinese Medicine. Vol. 42 (2010), p. 134-135

[2] Qiaoling Cai and Dayi Hu: The applications of Medical Simulation Technology in Clinical Teaching. Chinese Journal of Internal Medicine. Vol. 45 (2006), p. 357-358

[3] Lei Xue, Jianhua Li and Miqing Xu: The Research and Exploration of Medical Simulation Teaching Mode. China Higher Medical Education. Vol. 4 (2011), p. 72-73

[4] Nan Geng, Tianyuan Yu and Hui Liu: Cervical rotation pull localization operation characteristics analysis of the mechanical parameters. Journal of Changchun College of Traditional Chinese Medicine. Vol. 31 (2015), p. 607-610

[5] Liheng Wang, Shuchun Sun and Zhaohui Chen: Rigid Body Measurement of Chinese Manipulation Curing Cervical Spondylopathy. Chinese Journal of Traditional Medical Traumatology \& Orthopedics Vol. 17 (2009), p. 22-24

[6] Liguo Zhu, Minshan Feng and Fangshan Bi: Mechanical Measurement During Cervical Rotational Manipulation in Vivo. Chinese Journal of Rehabilitation Medicine. Vol. 22 (2007), p. 673-676

[7] Brekelmans WA, Poort HW, Slooff TJ. A new method to analysis the mechanical behavior of skeletal parts [J]. Acta Ortho Scand, 1972, 43(5): 301-317.

[8] Rybicki EF, Simonen FA, Weis EB Jr. On the mathematical analysis of stress in the human femur [J].J Biomech, 1972, 5(2): 203-215. 